\title{
Bases para el desarrollo e implementación de un modelo de información en salud de la mujer y perinatal orientado a la gestión en Latinoamérica
}

\author{
Ariel Karolinski, ${ }^{1}$ Raúl Mercer, ${ }^{2}$ Andrés Bolzán, ${ }^{3}$ Pablo Salgado, ${ }^{2}$ Celina Ocampo, ${ }^{3}$ \\ Ricardo Nieto, ${ }^{3}$ Maureen Birmingham, ${ }^{1}$ Gerardo Martínez, ${ }^{4}$ Luis Mainero, ${ }^{4}$ \\ Suzanne Serruya, ${ }^{4}$ Andrés De Francisco ${ }^{5}$ y Francisco Becerra-Posada ${ }^{5}$
}

Forma de citar

Karolinski A, Mercer R, Bolzán A, Salgado P, Ocampo C, Nieto R, et al. Bases para el desarrollo e implementación de un modelo de información en salud de la mujer y perinatal orientado a la gestión en Latinoamérica. Rev Panam Salud Publica. 2018;42:e148. https://doi.org/10.26633/RPSP.2018.148

RESUMEN Objetivo. El Sistema Informático Perinatal (SIP) ha marcado un hito en el uso de información sistematizada en la Región de las Américas. Lo que se ha aprendido ha contribuido al desarrollo de un modelo basado en un conjunto minimo de indicadores (CMI). El objetivo del estudio fue describir el proceso histórico y metodológico de desarrollo, implementación y escalamiento territorial de un CMI para monitorizar y evaluar políticas, programas y servicios de salud de la mujer y perinatal orientado a la gestión (SIP-GESTIÓN).

Métodos. El estudio se llevó a cabo en dos etapas: 1) validación en cuatro fases de un CMI en una red de hospitales: a) construcción del modelo teórico de indicadores, b) implementación de la investigación operativa, c) selección final de indicadores, y d) definición de patrones de referencia, y 2) escalamiento territorial.

Resultados. Se identificaron 17 modelos de indicadores. El modelo inicial incluyó 177 indicadores agrupados en siete dimensiones (contexto, hábitos, accesibilidad, uso de servicios, calidad de cuidados, impacto materno-fetal, e impacto materno-neonatal) que se redujeron a 21 tras tres rondas Delphi. El modelo final (SIP-GESTIÓN) incluyó 40 indicadores. Se analizaron 240 021 partos $(79,1 \%)$ de un total de 303559 atendidos en las 122 maternidades seleccionadas en 24 Jurisdicciones (100\%) de Argentina. La información se presenta a nivel nacional y desagregada por región sanitaria, provincia y hospital.

Conclusiones. Este modelo permitió alcanzar altos niveles de cobertura y calidad de la información y escalamiento territorial y es útil para la gestión, la investigación y la reorientación de programas y políticas.

Palabras clave Gestión de la información en salud; información científica; toma de decisiones en salud; sistemas de información; salud de la mujer; perinatología; Centro Latinoamericano de Perinatología; Salud de la Mujer y Reproductiva; Argentina.

\footnotetext{
Organización Panamericana de la Salud, Buenos Aires, Argentina. La correspondencia se debe dirigir a Ariel Karolinski. Correo electrónico: karolinskia@ paho.org (desde 2017 en la Organización Panamericana de la Salud, Caracas, Venezuela).
}

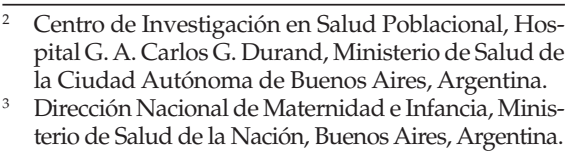

\footnotetext{
Centro Latinoamericano de Perinatología, Salud de la Mujer y Reproductiva, Montevideo, Uruguay.

Organización Panamericana de la Salud, Washington, DC, Estados Unidos de América.
} 
Durante las últimas décadas se han promovido a nivel global múltiples iniciativas para mejorar la salud de las mujeres y los niños desde el sistema de las agencias de Naciones Unidas y sus Estados. Un común denominador de estas propuestas es que se plantea disponer de sistemas de información adecuados que sirvan para contribuir, a través del análisis de datos, a obtener mejores resultados en salud. Actualmente, se reconoce la necesidad de integrar la información sobre salud de la mujer desde un enfoque de curso de vida, derechos, equidad, género, interculturalidad, intersectorialidad e interseccionalidad, con datos fiables sobre el contexto en que las mujeres llegan al embarazo, sus hábitos, la calidad de servicios de salud ofrecidos, el acceso a cuidados efectivos de salud sexual y salud reproductiva, y la evaluación del impacto maternofetal y neonatal (1-6).

La evaluación en salud de la mujer y perinatal debe disponer de modelos de información útiles para monitorizar los progresos en la capacidad de respuesta del sistema de atención de salud (7). Un sistema de indicadores sobre salud de la mujer y perinatal debe intentar mejorar tres aspectos: el ambiente, el empoderamiento de las usuarias y los resultados obtenidos (8-10).

El Sistema Informático Perinatal (SIP) desarrollado en 1983 por el Centro
Latinoamericano de Perinatología (CLAP), un centro especializado de la Organización Panamericana de la Salud/Organización Mundial de la Salud (OPS/OMS), marcó un hito en América Latina y el Caribe (ALC) para la gestión clínica del proceso reproductivo y se implementó en numerosos países de la Región de las Américas (11). En estos años se acumularon suficiente experiencia y evidencia sobre logros y limitaciones en el uso del SIP. Se hicieron importantes avances tanto en el desarrollo de nuevas funcionalidades tecnológicas para capturar información en línea como en nuevos módulos: "near miss", aborto, neonatal, defectos congénitos, comunitario, sífilis / VIH, y zika, entre otros (12-15). Pese a su reconocimiento regional, la información que proporciona el SIP no ha logrado aprovecharse debidamente para la gestión de políticas, programas y servicios de salud ni para la toma de decisiones fundamentada.

Este artículo tiene como objetivo describir el proceso histórico y metodológico para el desarrollo de un conjunto mínimo de indicadores (CMI) orientado a la gestión (SIP-GESTIÓN) y destinado a mejorar el uso de la información que este sistema proporciona, facilitar la monitorización y evaluación de políticas, programas, y servicios de salud de la mujer y perinatal, y promover estrategias de implementación y escalamiento regional de modelos desarrollados con este enfoque que respondan a las necesidades de los países.

\section{MATERIALES Y MÉTODOS}

La contribución del SIP-Gestión al SIP, basado en un CMI, fue el resultado de un proyecto dirigido al relevamiento (recolección y análisis de datos) epidemiológico, la capacitación y la investigación operativa mediante el uso sistemático del SIP en una red de 30 hospitales públicos del Área Metropolitana de Buenos Aires donde se atienden aproximadamente 90000 partos anuales ("Red Perinatal $\mathrm{AMBA}^{\prime \prime}$ ). Esta red está coordinada por el Centro de Investigación en Salud Poblacional (CISAP) del Ministerio de Salud de la Ciudad Autónoma de Buenos Aires $(16,17)$. La construcción del modelo final de indicadores propuesto se llevó a cabo en dos etapas: 1) validación de un CMI en una red de hospitales (18), y 2) escalamiento territorial.

La primera etapa estuvo integrada por las cuatro fases que se describen a continuación.

Fase 1. Construcción del modelo teórico de indicadores: se analizaron modelos de indicadores existentes a escala mundial mediante búsqueda de información secundaria de múltiples fuentes (18) (cuadro 1). Se identificaron y tipificaron indicadores según el marco teórico

\section{CUADRO 1. Modelo final del SIP-GESTIÓN: dimensiones, indicadores, definición y fuentes de información}

\begin{tabular}{|c|c|c|c|c|}
\hline Dimensión & & Indicadores & Definición & Fuente \\
\hline \multirow[t]{2}{*}{ Contexto } & 1 & Tasa de madres adolescentes (10-19 años) & Número de mujeres entre 10-19 años / Total de nacimientos & SIP \\
\hline & 2 & Tasa de madres $>35$ años & Número de mujeres > 35 años / Total de nacimientos & \\
\hline Hábitos & 3 & Tabaquismo materno & Número de mujeres que fumaron durante embarazo / Total nacimientos & \\
\hline \multirow[t]{2}{*}{ Accesibilidad } & 4 & Tasa de embarazos sin control prenatal & Número de mujeres sin control prenatal / Total de nacimientos & \\
\hline & 5 & $\begin{array}{l}\text { Tasa de embarazos con control prenatal } \\
\text { insuficiente }\end{array}$ & Número de mujeres con menos de 5 controles prenatales / Total de nacimientos & \\
\hline Uso de servicios & 6 & Tasa de cesáreas & Número de mujeres en las que se realizó cesárea / Total nacimientos & \\
\hline \multirow[t]{7}{*}{$\begin{array}{l}\text { Calidad de } \\
\text { los cuidados }\end{array}$} & 7 & Tasa de uso de corticoides en embarazo & $\begin{array}{l}\text { Número de mujeres que recibieron corticoides / Total nacimientos } \\
<\text { de } 35 \text { semanas }\end{array}$ & \\
\hline & 8 & Suplementación con hierro/folatos & Número de mujeres que recibieron hierro y/o folatos / Total nacimientos & \\
\hline & 9 & Acompañamiento en el parto & $\begin{array}{l}\text { Número de mujeres que estuvieron acompañadas en el parto / Total } \\
\text { nacimientos }\end{array}$ & \\
\hline & 10 & Manejo activo del alumbramiento & $\begin{array}{l}\text { Número de mujeres que recibieron oxitocina u otro oxitócico en } \\
\text { alumbramiento/ Total de mujeres con parto vaginal }\end{array}$ & \\
\hline & 11 & Episiotomía en primíparas & $\begin{array}{l}\text { Número de mujeres primíparas a las que se les realizó episiotomía / } \\
\text { Total de mujeres primíparas que tuvieron un parto vaginal no instrumental }\end{array}$ & \\
\hline & 12 & Sulfato de magnesio en eclampsia & $\begin{array}{l}\text { Número de mujeres que recibieron sulfato de magnesio / Total mujeres con } \\
\text { eclampsia }\end{array}$ & \\
\hline & 13 & Sulfato de magnesio en preeclampsia & $\begin{array}{l}\text { Número de mujeres que recibieron sulfato de magnesio / Total mujeres con } \\
\text { preeclampsia }\end{array}$ & \\
\hline
\end{tabular}


CUADRO 1. (Continuado)

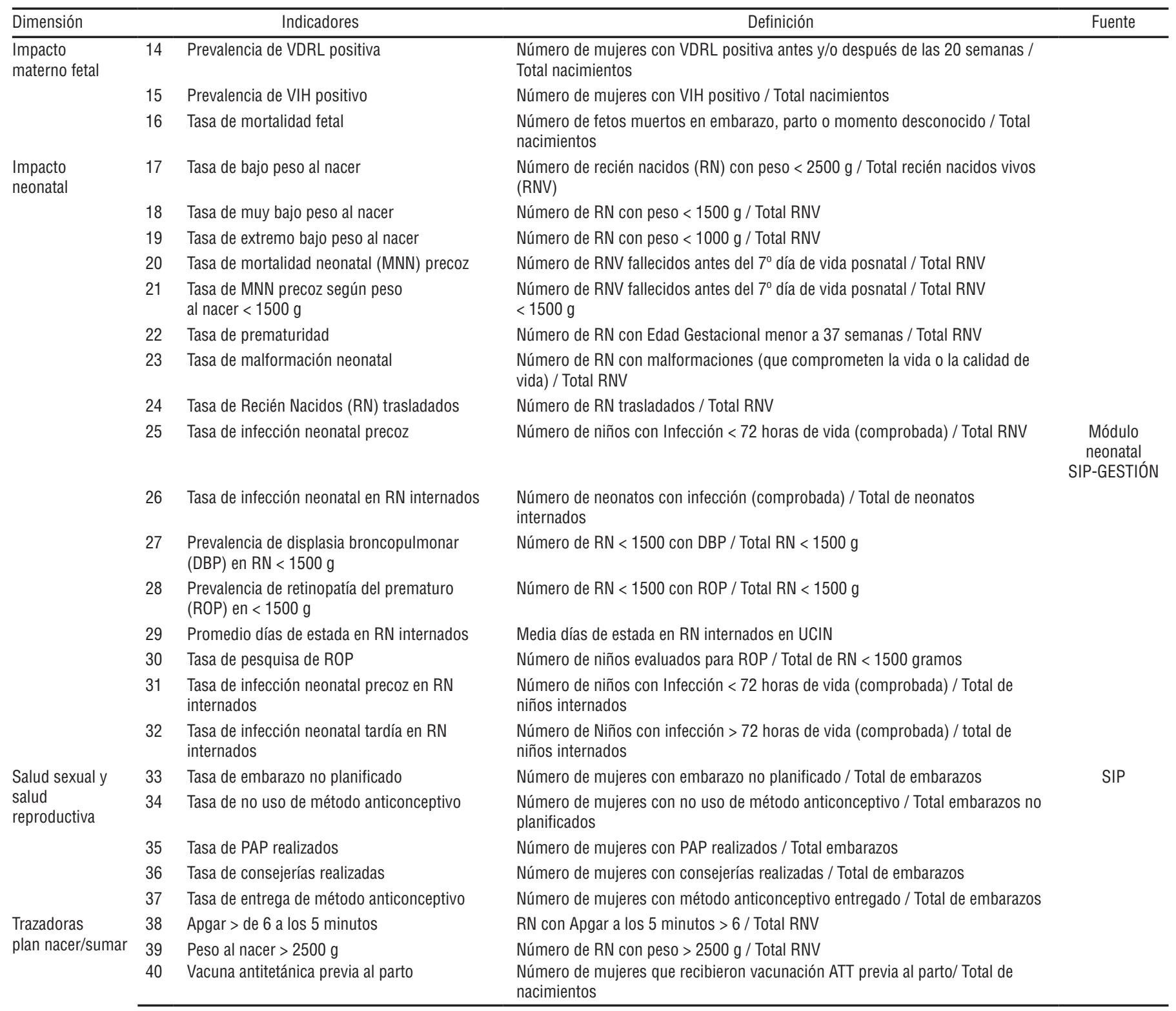

Nota: para los indicadores de calidad de cuidado consultar la Biblioteca de Salud Reproductiva de la OMS en https://extranet.who.int/rhl

adoptado y se clasificaron según diferentes criterios (ético, utilidad, robustez, representatividad, comprensibilidad, accesibilidad, significación clínica y epidemiológica) (19).

Fase 2. Implementación de la investigación operativa: se verificó la cobertura y la variación en el uso de prácticas perinatales beneficiosas correspondientes a indicadores de la dimensión "calidad de cuidados" (20) y se definió una línea basal para establecer tendencias (21).

Fase 3. Selección final de indicadores: mediante tres rondas de consulta (utilizando el método Delphi) varios jefes de servicio de obstetricia, especialistas en salud pública, tocoginecólogos, parteras profesionales y neonatólogos seleccionados de ocho hospitales públicos de la "Red Perinatal AMBA" que utilizan sistemáticamente el SIP (18, 22-23) asignaron puntuaciones a cada indicador y se calcularon las distribuciones de frecuencia, las medias y las desviaciones estándar. Los indicadores agrupados y desagregados se analizaron por las siguientes categorías: profesión, experiencia y tipo de hospital.
Con el ANOVA de una vía se compararon las medias de las puntuaciones obtenidas en cada indicador y las de las categorías. Se aplicaron pruebas estadísticas para muestras independientes y se fijó un nivel alfa de 5\%. En cada ronda de consultas se consideró como línea basal la media obtenida en cada indicador para ser incluido o excluido en la ronda siguiente. Para el análisis de datos de las fases 2 y 3 se usaron los programas Epi-Info 3.2.2 y Stata 9.0.

Fase 4. Definición de patrones de referencia: se utilizaron criterios de 
conveniencia (mejor evidencia disponible o mejor estándar logrado por los hospitales incluidos en la investigación) o el consenso de investigadores. La comparación de los resultados con el patrón de referencia contribuye al mejoramiento continuo del desempeño institucional (benchmarking) (24).

La segunda etapa se basó en la experiencia de la Red Perinatal AMBA/ CISAP y contó con la cooperación técnica de la OPS de Argentina y del Centro Latinoamericano de Perinatología-Salud de la Mujer y Reproductiva del Departamento de Salud Familiar, Promoción de la Salud y Curso de Vida (FPL) de la OPS (CLAP-SMR/FPL/OPS-OMS). El Ministerio de Salud de Argentina inició un proceso de escalamiento territorial a nivel nacional mediante la implementación del SIP, que incluyó el componente orientado a la gestión (SIP-GESTIÓN) (25). Para implementar esta propuesta se requirieron asociaciones estratégicas con diversas instituciones y la formación de grupos de discusión y consenso. (El lector interesado puede solicitar a los autores el material suplementario de este estudio.)

El modelo final de indicadores del SIPGESTIÓN aplicado a nivel nacional fue el resultado de la revisión de los indicadores originalmente incluidos en el CMI de la Red Perinatal AMBA, complementados con indicadores de salud sexual y reproductiva, un componente neonatal diseñado "ad hoc" (Módulo Neonatal del SIP-GESTIÓN), e indicadores trazadores del impacto del plan nacional de pago por prestaciones (PLAN Nacer/ SUMAR) (26). El modelo final se validó en una prueba piloto realizada en 15 maternidades representativas de diferentes regiones de Argentina. Para incluir los indicadores en el modelo final, cada indicador se evaluó según los siguientes criterios:

a) Cobertura (porcentaje de datos que faltan en el indicador) (27): excelente: $<5 \%$; muy buena: de $5 \%$ a $<10 \%$; buena: de $10 \%$ a $<15 \%$; regular: de $15 \%$ a $<20 \%$; mala: de $20 \%$ a $<30 \%$, e inaceptable: $\geq 30 \%$.

b) Dificultad de captura del dato (basado en el porcentaje de hospitales con cobertura mala o inaceptable del indicador): alta: entre 51 y 100\%; media: entre 21 y $50 \%$, y baja: entre 0 y $20 \%$.

c) Factibilidad resolutiva de la dificultad de captura del dato: se incluye o se excluye según la facilidad o dificultad en la resolución.

d) Indicador único o "proxy": hay un solo indicador para evaluar la dimensión o es un "proxy" (más de un indicador evalúa la misma dimensión).

e) Decisión final de inclusión: se incluye o se elimina de la lista.

Cuando la cobertura general de información de un hospital fue menor de $70 \%$, éste se excluyó del análisis.

Los resultados se analizaron desde dos perspectivas. Primera, de base poblacional, donde la unidad de análisis fue individual (mujer, feto o neonato). Para ello se trabajó con una base de datos consolidada correspondiente a todos los hospitales incluidos. Segunda, de base hospitalaria, donde la unidad de análisis fue el hospital. Para ello se trabajó comparando las bases individuales de cada establecimiento.

En los análisis se calcularon valores absolutos, porcentajes, con sus correspondientes intervalos de confianza de 95\% (IC95\%), medianas y rangos intercuartílicos. Para caracterizar los indicadores seleccionados se establecieron dos criterios de variabilidad: rango y brecha (diferencia y cociente entre valores extremos, respectivamente).

Para realizar el análisis de base poblacional se construyeron series temporales de cada indicador con su respectivo patrón de referencia y el criterio utilizado para su formulación (28). Para el análisis de base hospitalaria se presentan gráficos de barras para cada hospital y para las medidas resumen de nivel nacional, regional o provincial, según corresponda. En los denominadores de los indicadores se incluyeron todos los nacimientos o embarazadas o mujeres según el atributo vinculado con la forma de finalización del embarazo o la presencia de algún factor de riesgo asociado. Para analizar los datos se utilizaron los paquetes estadísticos SIP 2007, EPI-INFO 3.2.2, Epidat 3.1, R Project 2.14.2, R Commander 1.8-3 y SPSS 20.

La identidad de cada hospital fue preservada y codificada a fin de garantizar la confidencialidad de la información. El código de identidad hospitalaria era conocido por cada institución a efectos del análisis comparativo. Asimismo, se preservó el secreto estadístico de información personal al usar las bases de datos.

\section{RESULTADOS}

\section{Validación de un CMI en una red de hospitales (Red Perinatal AMBA)}

Se identificaron 17 modelos de indicadores propuestos por diferentes agencias y organismos (material suplementario). El modelo inicial incluyó 177 indicadores y se redujo progresivamente a 79, 48 y, finalmente, a 21 tras completar tres rondas Delphi. Los indicadores se agruparon en siete dimensiones: contexto (dos), hábitos (uno), accesibilidad (uno), uso de servicios (uno), calidad de cuidados (siete), impacto materno-fetal (cuatro) e impacto materno-neonatal (cinco).

\section{Escalamiento territorial: modelo final de indicadores del SIP- GESTIÓN a nivel nacional}

El modelo final incluyó 40 indicadores según el consenso de los expertos. De los 21 indicadores originales se excluyeron cuatro. A los 17 agrupados en las siete dimensiones ya referidas se añadieron uno de contexto, uno de accesibilidad, 13 de impacto neonatal, cinco de salud sexual y reproductiva, y tres indicadores trazadores del impacto del PLAN Nacer/ SUMAR. El SIP fue la fuente de información para 32 indicadores y el módulo neonatal del SIP-GESTION aportó los ocho indicadores restantes (cuadro 1). La definición, la construcción, el procesamiento y el análisis de cada uno de los indicadores seleccionados se describe en el Manual Operativo del SIP-GESTIÓN (28).

Se analizaron 240021 partos $(79,1 \%)$ de un total de 303559 partos atendidos en 122 maternidades seleccionadas en las 24 jurisdicciones político-administrativas de Argentina (23 provincias y Ciudad Autónoma de Buenos Aires). La información se presenta a escala nacional y desagregada por región sanitaria, provincia y hospital.

El cuadro 2 muestra los resultados principales de los indicadores seleccionados del SIP-GESTIÓN a nivel nacional. A modo de ejemplo, se presentan resultados de variación a nivel regional, provincial y hospitalario del indicador de uso de servicios proporción de cesáreas. Se incluyen gráficos de barras con la proporción del indicador para el total del país y para cada región, provincia y hospital considerados y en ellos se detallan las medidas de variación estimadas 
CUADRO 2. Resultados principales de los indicadores seleccionados del SIP-GESTION por dimensión, Argentina, 2011

\begin{tabular}{|c|c|c|c|c|c|c|c|}
\hline Indicador & Porcentaje & $\mathrm{IC} 95 \%$ & $\begin{array}{c}\text { Datos perdidos } \\
(\%)\end{array}$ & $\begin{array}{l}\text { Calidad de } \\
\text { cobertura }\end{array}$ & $\begin{array}{l}\text { Dificultad en } \\
\text { captura }\end{array}$ & $\begin{array}{c}\text { Hospitales excluidos } \\
\text { (No.) }\end{array}$ & $\begin{array}{l}\text { Hospitales incluidos } \\
(\%)\end{array}$ \\
\hline \multicolumn{8}{|c|}{ Contexto } \\
\hline Madre adolescente & 23,7 & $23,5-23,9$ & 0,5 & Excelente & Baja & 0 & 100 \\
\hline Madre < 15 años & 0,8 & $0,76-0,84$ & & & & & \\
\hline Madre > 35 años & 8,1 & $8,0-8,2$ & & & & & \\
\hline \multicolumn{8}{|c|}{ Hábitos } \\
\hline Tabaquismo & 10,1 & $10,0-10,3$ & 7,1 & Muy buena & Baja & 13 & 88,5 \\
\hline \multicolumn{8}{|c|}{ Accesibilidad } \\
\hline Sin control prenatal & 6,9 & $6,8-7,1$ & 7,5 & Muy buena & Baja & 7 & 93,8 \\
\hline Control prenatal insuficiente & 33,5 & $33,3-33,8$ & & & & & \\
\hline \multicolumn{8}{|c|}{ Uso de servicio } \\
\hline Tasa de cesáreas & 29,1 & $28,9-29,3$ & 1,8 & Excelente & Baja & 0 & 100 \\
\hline \multicolumn{8}{|c|}{ Calidad de cuidado } \\
\hline Corticoides prenatales & 45,4 & $44,3-46,5$ & 8,1 & Muy buena & Media & 33 & 70,8 \\
\hline Suplementación con Fe y folatos & 72,9 & $72,6-73,1$ & 9,6 & Muy buena & Alta & 58 & 48,7 \\
\hline Acompañamiento en el parto & 34,2 & $33,9-34,5$ & 7,7 & Muy buena & Alta & 53 & 53,1 \\
\hline Manejo activo del alumbramiento & 71,8 & $71,6-72,1$ & 11,2 & Buena & Media & 25 & 77,9 \\
\hline Episiotomía en primíparas & 62,5 & $62,0-63,0$ & 8,0 & Muy buena & Media & 21 & 81,4 \\
\hline Sulfato de Mg en preeclampsia & 14,4 & $11,6-17,7$ & 5,6 & Muy buena & Alta & 108 & 4,4 \\
\hline Sulfato de Mg en eclampsia & 53,5 & $37,8-68,5$ & 14,0 & Buena & Alta & 107 & 5,3 \\
\hline \multicolumn{8}{|c|}{ Impacto maternofetal } \\
\hline Prevalencia de VDRL + & 1,4 & $1,31-1,41$ & 7,1 & Muy buena & Media & 24 & 78,8 \\
\hline Tasa de mortalidad fetal & $7,7 \%$ & $7,4 \% 0-8,1 \%$ & 0,4 & Excelente & Ваја & 0 & 100 \\
\hline Prevalencia de HIV+ en madres & 0,4 & $0,35-0,51$ & 12,1 & Buena & Alta & 103 & 8,8 \\
\hline \multicolumn{8}{|c|}{ Impacto neonatal } \\
\hline Tasa de prematuridad & 10,6 & $10,4-10,7$ & 0 & Excelente & Baja & 0 & 100 \\
\hline Tasa de BPN & 8,2 & $8,1-8,4$ & 0 & Excelente & Baja & 0 & 100 \\
\hline Tasa muy BPN < $1500 \mathrm{~g}$ & 1,7 & $1,6-1,8$ & 0 & & & & \\
\hline Tasa extremo BPN < $1000 \mathrm{~g}$ & 0,7 & $0,67-0,74$ & 0 & & & & \\
\hline RN trasladados & 0,7 & $0,6-0,7$ & 12,4 & Buena & Media & 40 & 64,6 \\
\hline Malformación neonatal & 0,8 & $0,8-0,9$ & 15,0 & Regular & Alta & 62 & 45,1 \\
\hline \multicolumn{8}{|c|}{ Salud sexual y salud reproductiva } \\
\hline Embarazo no planificado & 61,5 & $61,3-61,7$ & 10,5 & Buena & Media & 29 & 74,3 \\
\hline Tasa no uso de MAC & 80,1 & $79,8-80,3$ & 11,2 & Buena & Alta & 51 & 54,9 \\
\hline Tasa de PAP realizados & 65,7 & $65,4-66,0$ & 10,8 & Buena & Alta & 59 & 47,8 \\
\hline Tasa de consejerías realizadas & 98,8 & $98,7-98,8$ & 8,8 & Muy buena & Media & 22 & 80,5 \\
\hline Tasa entrega de MAC & 91,5 & $91,4-91,7$ & 9,5 & Muy buena & Media & 40 & 64,6 \\
\hline \multicolumn{8}{|c|}{ Trazadoras del Plan Nacer/SUMAR } \\
\hline Apgar $>6$ a los 5 minutos & 98,5 & $98,4-98,6$ & 1,3 & Excelente & Baja & 0 & 100 \\
\hline Peso al Nacer $>2500 \mathrm{~g}$ & 91,8 & $91,6-91,9$ & 0 & Excelente & Baja & 0 & 100 \\
\hline Vacuna ATT previa al parto & 86,8 & $86,7-87,0$ & 7,8 & Buena & Media & 10 & 91,2 \\
\hline
\end{tabular}

(brecha y rango) (figura 1). Finalmente, se presentan las tendencias en el uso de indicadores seleccionados con sus patrones de referencia para el período 20102013 (figura 2).

\section{Resultados de la cooperación técnica con la OPS/CLAP-SMR}

El proceso de desarrollo e implementación del SIP-GESTIÓN requirió un espacio de intercambio técnico entre los equipos profesionales nacionales del SIPGESTIÓN, de la OPS de Argentina y del
CLAP-SMR/FPL/OPS-OMS. El equipo de SIP-GESTION y de la OPS de Argentina presentó los contextos y los indicadores por dimensiones surgidos del proceso narrado. El equipo técnico de CLAP-SMR desarrolló la sintaxis de los indicadores solicitados y generó una nueva salida automática del grupo de indicadores "BÁSICOS" ("one click") existentes en el SIP, que se denominó SIP-GESTION. Esta opción de análisis fue preparada para que esta funcionalidad se visualizara $y$ ejecutara sólo cuando el programa fuera configurado para Argentina.
Este desarrollo obligó a realizar ajustes en el programa, incorporar nuevas variables originalmente no contempladas en la versión de consenso regional en uso y crear programas especiales para definir variables de interés en el modelo de llenado obligatorio. También se corrigieron errores circunstanciales del programa durante la etapa de producción de la nueva funcionalidad SIP-GESTION. La comunicación y el intercambio entre todos los participantes fueron permanentes durante todo el proceso. 
FIGURA 1. Dimensión uso de sevicios. Porcentaje de cesáreas. SIP-GESTIÓN, Argentina, 2011
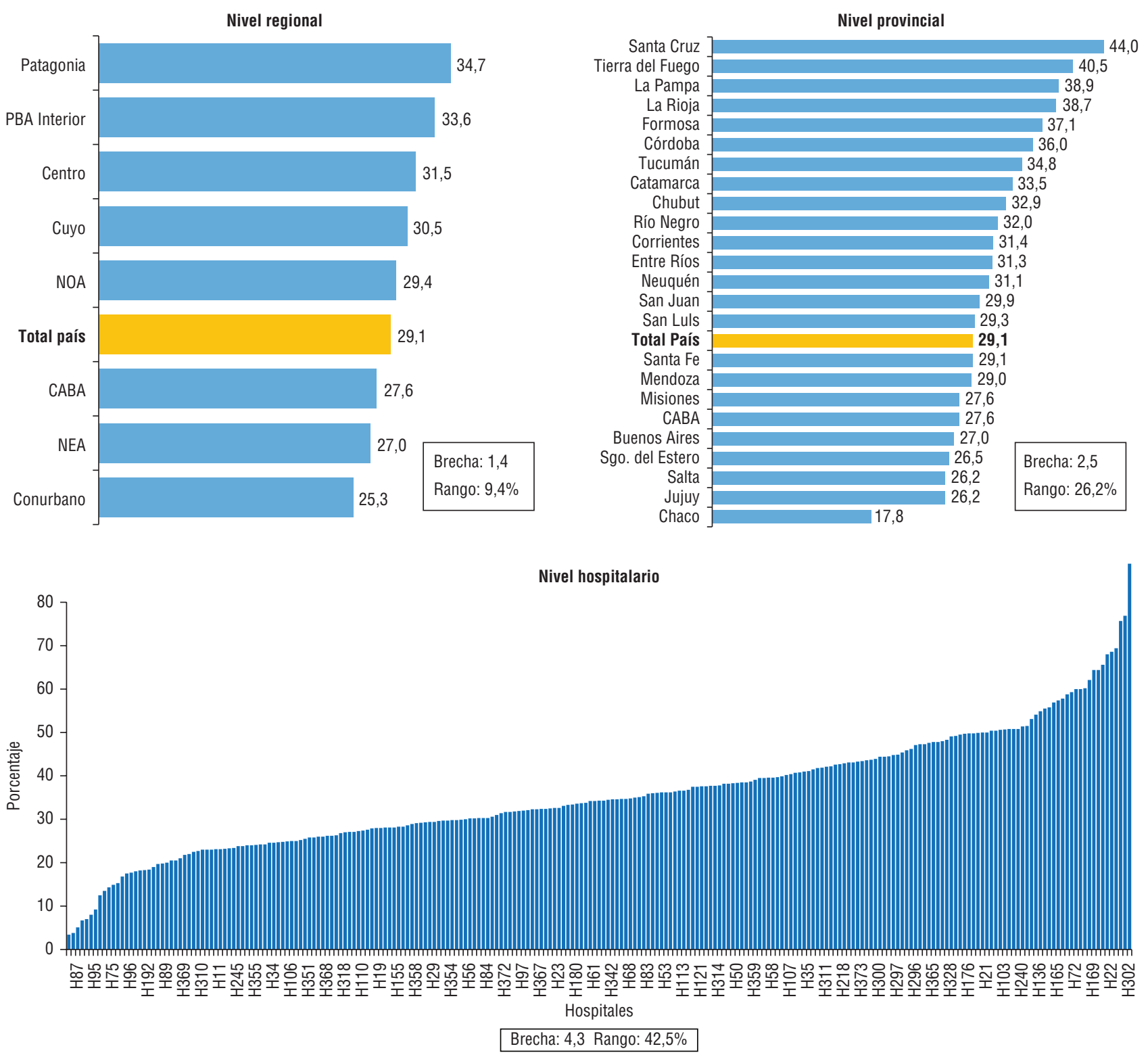

Cuando la nueva funcionalidad se incorporó y estaba operativa, el SIP 2007 ofreció las opciones de análisis de indicadores seleccionados del SIP-GESTIÓN. Para mejorar la cobertura de las variables del CMI del SIP-GESTIÓN, el equipo técnico de CLAP-SMR desarrolló un programa que permite configurar dichas variables como obligatorias (no permite guardar la historia clínica si no se tiene el dato de cada una de ellas) o recomendadas (permite guardar la historia clínica, pero previamente el sistema emite una alerta para su correcto llenado). Además, este proceso de selección de variables necesarias permitió recalcular la cobertura de control de llenado (material suplementario).

\section{DISCUSIÓN}

Este artículo describe el proceso metodológico de desarrollo de un conjunto de 40 indicadores para el monitoreo y la evaluación de políticas, programas, y servicios de salud de la mujer y perinatal, utilizando como fuente primaria de datos el SIP/OPS-OMS. Presenta una modalidad de análisis de resultados considerando la variabilidad poblacional e institucional de los indicadores y su estandarización, aporta lecciones aprendidas para el escalamiento territorial a nivel nacional destacando la importancia de los modelos asociativos entre el Estado, agencias de cooperación e instituciones de investigación, y, finalmente, plantea la posibilidad de extender la propuesta del SIP-GESTIÓN a nivel Regional como actualmente lo realiza CLAP-SMR/FPL/OPS-OMS.

La primera etapa de construcción del modelo por el CISAP/Red Perinatal AMBA permitió identificar un CMI de 21 indicadores a partir del análisis y las rondas Delphi basados en dos estudios multicéntricos $(18,20)$ y los indicadores básicos del SIP. Se analizaron modelos 
FIGURA 2. Tendencias de las tasas de uso de indicadores seleccionados con sus patrones de referencia, SIP-GESTIÓN, Argentina, 2010-2013
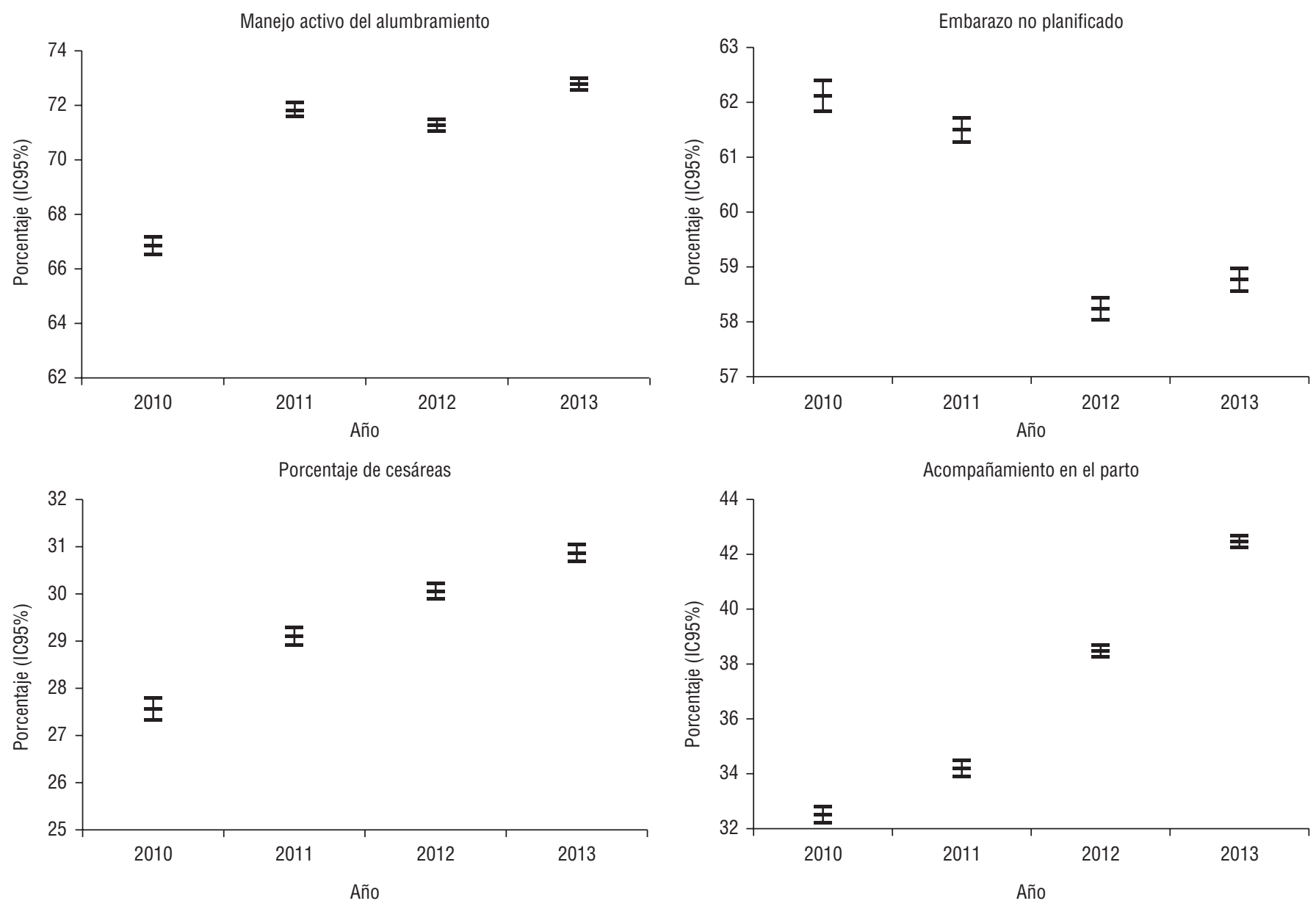

teóricos basados en determinantes de la salud, procesos de atención y resultados $\mathrm{y}$ factores contextuales propuestos por el modelo de determinantes múltiples (29-42). Los indicadores seleccionados caracterizan contextos del proceso reproductivo, hábitos de las mujeres y factores asociados con el proceso de atención, y describen acontecimientos durante el período perinatal y el peso relativo de los momentos (prenatal, periparto y postnatal). Los indicadores de resultado fueron los que mayor puntuación recibieron y mayor frecuencia relativa alcanzaron. Algunos indicadores basados en la opinión de las usuarias se descartaron, lo que indica que la opinión de las mujeres no es un factor relevante para los prestadores encuestados. Se deberán fortalecer acciones para que los equipos de salud valoren la percepción de las usuarias aplicando encuestas periódicas. El papel y la experiencia profesionales mostraron heterogeneidad. La influencia de ambos factores sobre la selección final de indicadores debe considerarse en la definición del CMI.

El SIP-GESTIÓN debe contextualizarse a la realidad de cada país y resultar del acuerdo con las autoridades sanitarias sobre la viabilidad del modelo. Para potenciar el papel de rectoría del Estado, la herramienta debe incluir información completa de todos los entes prestadores de servicios (público, seguridad social y privado). El sistema de indicadores del SIP-Gestión contribuye a fortalecer la regionalización sanitaria, potenciar la conformación de redes de servicios, facilitar la evaluación y acreditación de servicios de salud perinatales, y ofrece un mecanismo de rendición de cuentas.

El SIP-GESTIÓN facilitó la articulación de los diferentes programas y planes que abordan la salud de la mujer y perinatal. Además, responde a una lógica del uso eficiente de la información bajo el criterio "una fuente de información para múltiples destinatarios" (material suplementario). Con él se busca optimizar recursos, evitar duplicaciones y promover una fuente de información que pueda ser modificada y actualizada en función de las necesidades o de los cambios de los perfiles epidemiológicos. En este sentido, el CLAP-SMR/FPL/OPSOMS ya ha incorporado esta funcionalidad atendiendo a lo solicitado por los países, como en Uruguay, a través de la experiencia del SIP-METAS, teniendo en cuenta los desarrollos realizados en Nicaragua, Bahamas, Trinidad y Tobago, y la está iniciando en la República Dominicana y en Panamá.

El modelo propuesto debe someterse a revisión y evaluación periódicas. Por ejemplo, varios indicadores de otras dimensiones incluidas podrían ser utilizados como indicadores de uso de servicios. La tasa de cesáreas permite evaluar múltiples aspectos del proceso de atención de servicios de salud 
materno-perinatales. Conociendo la heterogeneidad regional referente a la sobreutilización y subutilización de esta intervención, estimar su tasa permite evaluar el perfil y el grado de adecuación de las prácticas de cada institución. Ello no es óbice para que en el futuro, y tras la aplicación sistemática del modelo, surja la necesidad de incorporar o cambiar alguno de los indicadores. El análisis de tendencias de los indicadores, la adecuación e incorporación de nuevas variables y el desarrollo de aplicativos orientados a fortalecer el uso de intervenciones beneficiosas para la salud de la mujer y perinatal conforman algunas de las líneas en desarrollo. Así, el CLAP-SMR/FPL/OPS-OMS promueve el uso de sistemas de carga en línea (SIP WEB, SIP para Tablet y telefonía móvil), la implementación de los módulos "near miss" para vigilancia de la morbilidad materna grave y SIP aborto $(12,13)$ y la aplicación de la clasificación de Robson como estrategia para controlar el aumento de las cesáreas en la Región.

El SIP-GESTIÓN es una herramienta potente para llevar a cabo investigaciones operativas y para la gestión del conocimiento, con él se pueden analizar variaciones en el uso de intervenciones para la mejora continua de calidad de atención de servicios de salud perinatal (20; 43-45), aporta información clave para la estrategia de control y erradicación de sífilis materna y congénita (13), y puede utilizarse sistemáticamente en redes colaborativas de investigación (Red CLAP y Red MUSA) (12).

Frecuentemente, la información se recolecta de manera sistemática, pero rara vez se utiliza para promover cambios asistenciales. Esta propuesta no se limita exclusivamente a recolectar información, sino que promueve cambios en la realidad asistencial hospitalaria, así como en programas y políticas sanitarias de cada país.

La existencia de marcos normativos o legales para utilizar el SIP no es garantía suficiente para su cumplimiento ni para el uso de la información generada. Un desafío estriba en buscar alternativas resolutivas tanto desde la implementación de políticas públicas como desde la adecuación de modalidades de prestación de servicios. A diferencia del SIP, ampliamente difundido y culturalmente aceptado en la mayoría de las maternidades públicas de Argentina y disponiendo de normas nacionales y provinciales que regulan su uso en forma obligatoria (46), el SIP NEONATAL tiene un desarrollo temporal mucho más reciente en Argentina y se utiliza en pocos servicios de neonatología. Ante la necesidad urgente de disponer de una herramienta útil y de fácil implementación, se desarrolló un programa operativo "ad hoc", que incluyó variables e indicadores definidos por consenso de expertos neonatólogos. Se diseñó una planilla de carga en papel que se completa al egreso de la unidad neonatal con posibilidad de exportar las planillas a Excel o a un programa estadístico. Este desarrollo compuesto por planilla y programa se denominó Módulo Neonatal SIP-GESTIÓN y permite calcular indicadores neonatales de resultado, de calidad de la atención y de uso de servicios.

Una preocupación importante de esta propuesta es lograr niveles de autonomía crecientes de los profesionales en el uso racional de información para la toma de decisiones en establecimientos asistenciales. Un sistema de información es más que un conglomerado de datos. Es una herramienta articuladora que motiva a los equipos locales a ser artífices de sus propios destinos institucionales a partir del conocimiento real, confiable y crítico de sus propias realidades. Además, contribuye a generar un espacio de debate para evaluar la capacidad de respuesta y la contribución real de los servicios de salud para la mejora de la salud de la mujer y perinatal, redefiniendo papeles y competencias institucionales dentro de un sistema asistencial regionalizado.

La generación de una cultura de la información requiere tiempo para ser efectiva. Se debe promover un uso democrático y responsable de la información en salud de la mujer y perinatal en todos los niveles de decisión como seguro y respaldo de acciones que impulsen los Estados para el logro de sus metas en salud.

El desarrollo e implementación del SIP-GESTIÓN debe apoyarse en acciones que aseguren su sostenibilidad, promuevan una mejora continua del modelo, permitan evaluar objetivamente el grado de uso de la información para la toma de decisiones, así como comprobar los cambios acontecidos en la gestión de programas y la provisión de servicios. La finalidad es contribuir a mejorar la salud de la mujer y perinatal.
El desarrollo del SIP-GESTIÓN como modelo general y su aplicación en campos específicos de la salud de la mujer y perinatal está adquiriendo un efecto demostrativo a partir de experiencias de implementación ya mencionadas en varios países de la Región, además de lo expuesto en este artículo para Argentina. Para traducir esta propuesta en un proceso de cambio real son necesarios varios factores catalizadores: mantener el SIP como un estándar regional que interpreta las realidades nacionales y locales para hacer posible un proceso de escalamiento territorial eficiente, y contar con la voluntad política y los recursos necesarios para su implementación. Algunas ventajas comparativas del SIP son su gratuidad, el hecho de que se acompañe del soporte técnico del CLAP-SMR/FPL/OPS-OMS y la posibilidad de exportar sus datos y ser compatible con otros sistemas de información sanitaria usados en los países. Esta condición de interoperabilidad del SIP lo sitúa como una herramienta esencial en información de salud de la mujer y perinatal para evaluar iniciativas orientadas a garantizar la cobertura y el acceso universales.

Es fundamental promover la cooperación entre países a través de las representaciones de la OPS/OMS y el CLAP-SMR/FPL/OPS-OMS, lo que históricamente ha constituido una acción esencial para avanzar en esta propuesta nacida en 1983 que continúa vigente gracias al esfuerzo de los países y los usuarios. Esto se estimula en la medida en que se gana apropiación, experiencia y confianza para incorporar nuevas herramientas tecnológicas.

El SIP-GESTIÓN es una contribución más, aunque no la única, que el CLAP-SMR, el Departamento FPL y las representaciones de la OPS/OMS de los países están promoviendo conjuntamente en toda la Región. Se trata de la puesta en marcha de una funcionalidad que ayude no sólo a mejorar las capacidades de equipos de salud responsables de programas y gestores de políticas en el mejor conocimiento y manejo de sus realidades relacionadas con salud de la mujer y perinatal, sino, fundamentalmente, a contribuir genuinamente al logro de la reducción de la morbimortalidad materna e infantil en la Región.

Agradecimientos. Los autores expresan su agradecimiento a los participantes 
de los Grupos de Discusión y Consenso para la formulación del SIP-GESTIÓN enumerados a continuación:

Grupo coordinador: Dr. Ariel Karolinski, Dr. Raúl Mercer, Lic. Pablo Salgado, Dra. Celina Ocampo, Lic. Andrés Bolzán (equipo asesor científico-técnico de profesionales del CISAP), Dr. Daniel Lipchak, Lic. Obst. Carolina Nigri (representantes de Obstetricia), Dra. Isabel Kurlat, Dr. Ricardo Nieto (Neonatología), Lic. Celina Sand (SIP), y Lic. Pablo Cadoche, Lic. Juan Schifres (Informática) de la Dirección Nacional de Maternidad e Infancia del Ministerio de Salud de la Nación, Argentina (Dra. Ana Speranza, Directora).
Grupo de Obstetricia: Dra. Analía Messina (Htal. Álvarez), Dra. Cristina Laterra (Maternidad Sardá), Dr. Mario Palermo (Hospital Posadas), Dr. Raúl Winograd (Hospital Argerich), Dr. Miguel Huespe (Hospital Santojanni), Dr. Daniel Fernández (Maternidad de San Isidro)

Grupo de Salud Sexual y Salud Reproductiva: Dra. Edith Pantelides (CENEP), Dr. Sergio Maulen y Lic. Eleonor Faur (UNFPA), Dr. Luis Roberto Escoto (OPS/ OMS -Representación Argentina-), y Dra. Suzanne Serruya y Dr. Bremen de Mucio (CLAP-SMR).

Grupo de Neonatología: Dra. Susana Rodríguez (Hospital Garrahan), Dra.

\section{REFERENCIAS}

1. Naciones Unidas. Resolución $55 / 2$ de la Asamblea General. Declaración del Milenio, 13 de septiembre de 2000. Nueva York: Naciones Unidas; 2000. Disponible en: http://www.un.org/ spanish/milenio/ares552.pdf Acceso el 14 de abril de 2018.

2. Naciones Unidas. Proyecto de documento final de la cumbre de las Naciones Unidas para la aprobación de la agenda para el desarrollo después de 2015. Nueva York: Naciones Unidas; 2015. Disponible en: http://www.un.org/ es/comun/docs/?symbol=A/69/L.85 Acceso el 14 de abril de 2018.

3. Naciones Unidas. Todas las Mujeres, todos los niños. Estrategia mundial para la salud de la mujer, el niño y el adolescente (20162030). Nueva York: Naciones Unidas; 2015. Disponible en: http://www.who.int/ maternal_child_adolescent/documents / women-deliver-global-strategy / es / Acceso el 14 de abril de 2018.

4. Organización Panamericana de la Salud. Plan estratégico de la Organización Panamericana de la Salud 2014-2019. “En pro de la salud: Desarrollo sostenible y equidad". Washington, DC: OPS; 2013. Disponible en: http://iris.paho.org/xmlui/ bitstream/handle/123456789/4034/ OD345-s.pdf? sequence $=1$ \&isAllowed $=y$ Acceso el 14 de abril de 2018.

5. Commission on information and accountability for women's and children's health. Keeping promises, measuring results. Geneva: World Health Organization; 2011. Disponible en: http://www.everywomaneverychild.org/images/content/files/ accountability_commission/final_report/ Final_EN_Web.pdf Acceso el 14 de abril de 2018.

6. Centro Latinoamericano de Perinatología, Salud de la Mujer y Reproductiva. Plan de acción para acelerar la reducción de la MM y la morbilidad materna grave. Montevideo: CLAP/SMR; 2012. Publicación Científica 1590. Disponible en: http://www. paho.org $/$ clap $/$ index.php?option $=\mathrm{com}_{-}$ content\&view=article\&id=167:plan-deaccion-para-acelerar-la-reduccion-de-lamortalidad-materna-y-la-morbilidad-materna-grave \&Itemid=234\&lang=es Acceso el 14 de abril de 2018.

7. Campbell SM, Cantrill JA, Roberts D. Prescribing indicators UK General Practice. Delphi consultation study. BMJ. 2000;321:425-8.

8. Health Canada. Canadian Perinatal Health Report, 2003. Ottawa: Minister of Public Works and Government Services Canada; 2003.

9. Dawn P, Guyer B, Allston A. Integrated Perinatal Framework. A multiple determinants model with a life span approach. Am J Pev Med. 2003;25(1):67-75.

10. Devane D, Begley C, Boyle C. Safety and maternity care: Identification of a core set of outcome measures using an e-Delphi process. Dublin: School of Nursing and Midwifery Studies, Trinity College; 2005.

11 Schwarcz R, Díaz AG, Fescina R, Díaz JL, Martell M, Simini F. The Perinatal Information System I: the simplified perinatal clinical record. J Perinat Med. 1987;15(Suppl 1):9.

12. Centro Latinoamericano de Perinatología, Salud de la Mujer y Reproductiva (CLAP/SMR). Reunión "Salvando mujeres y recién nacidos: intervenciones para reducir la mortalidad materna y neonatal". Panamá, 14 al 16 de septiembre de 2015. Disponible: http://www.paho.org/ clap/index.php?option=com_content\& view $=$ article $\& i d=332$ : ultimos-avancesdel-sip-en-la-region\&Itemid=215\&lang= es Acceso el 14 de abril de 2018.

13. Karolinski A, Mercer R, Bolzán A. Proyecto Investigación operativa Utilidad del Sistema Informático Perinatal (SIP): gestión para la vigilancia epidemiológica de la Sífilis materna y sífilis congénita en una red de hospitales públicos del área metropolitana de Buenos Aires, Argentina: informe final. Montevideo: CLAP/SMR; 2012. (CLAP/SMR. Publicación científica; 1592). ISBN: 978-92-75-31686-3. Disponible
Regina Valverde (Maternidad de San Isidro), Dr. Carlos Grandi (Maternidad Sardá), Dr. Alberto Schwarcz (Provincia de Bs. As. Región Sanitaria V), y Dr. Nestor Vain (sector privado).

Financiación. Este estudio no ha recibido financiación.

Conflictos de interés. Los autores declaran no tener conflictos de interés.

Declaración. Las opiniones expresadas en este manuscrito son responsabilidad del autor y no reflejan necesariamente los criterios ni la política de la RPSP/ PAJPH y/o de la OPS. en: http://www.paho.org/clap/index.php? option $=$ com_docman\&view $=$ docum ent\&Itemid $=219 \&$ la yout $=$ default $\&$ alias $=288$-utilidad-del-sip-gestion-parala-vigilancia-epidemiologica-de-la-sifilismaterna-y-sifilis-congenita-en-argentina$5 \&$ category_slug=sip\&lang=es Acceso el 14 de abril de 2018.

14. Chaves SC, Cecatti JG, Carroli G, Lumbiganon P, Hogue CJ, Mori R, et al. Obstetric transition in the World Health Organization Multicountry Survey on Maternal and Newborn Health: exploring pathways for maternal mortality reduction. Rev Panam Salud Publica. 2015;37(4/5):203-10.

15. Karolinski A, Mercer R, Micone P, Ocampo C, Salgado P, Szulik D, et al. Modelo para abordar integralmente la mortalidad materna y la morbilidad materna grave. Rev Panam Salud Publica. 2015;37(4/5):351-9.

16. Karolinski A, Mercer R, Micone P, Mazzoni A, Wainer V, Sanchez A, et al. Proyecto red de centros perinatales del área metropolitana de Buenos Aires y sur de la provincia de Buenos Aires Red Perinatal AMBA: tres años de gestión asociada. Revista HD (Publicación Científica del Hospital Durand). 2007;5(3);2007:95-101.

17. Karolinski A, Mercer R, Micone P, Mazzoni A, Bolzán A, Wainer V, et al. Importancia del uso de indicadores epidemiológicos para mejorar la calidad de atención en salud materna y perinatal en hospitales públicos de la Argentina: la experiencia de la Red Perinatal AMBA. Proago: Programa de Actualización en Ginecología y Obstetricia. Editorial Panamericana; 2008, Duodécimo Ciclo, Módulo 1:155-215.

18. Bolzán A. Validación de un modelo para evaluar progresos en salud materno perinatal en establecimientos públicos de la Provincia de Buenos Aires. Informe final Beca CONAPRIS 2006/2007. Buenos Aires, Argentina: Ministerio de Salud de la Nación; 2007.

19. World Health Organization. Monitoring reproductive health: Selecting a short list 
of national and global indicators. Geneva: WHO; 1997.

20. Karolinski A, Micone P, Mercer R, Gibbons L, Althabe F, Belizán JM, et al. Evidencebased maternal and perinatal healthcare practices in public hospitals in Argentina. Int J Gynaecol Obstet. 2009;105(2):118-22. Disponible en: https://www.ncbi.nlm. nih.gov/pubmed/19232607 Acceso el 14 de abril de 2018.

21. Karolinski A, Mercer R, Bolzán A, Micone $\mathrm{P}$, Mazzoni $\mathrm{A}$, Wainer $\mathrm{V}$, et al. $4^{\circ}$ Informe de relevamiento epidemiológico del proyecto "Red Perinatal AMBA" 2004/2005/2006/2007. Buenos Aires: Centro de Investigación en Salud Poblacional; 2009. (Documento Técnico No. 01/2009.)

22. Hanafin S, Brooks AM. The Delphi Technique. A methodology to support the development of a National set of child well-being indicators. Dublin: The National Children Office; 2005.

23. Gupta UG, Clarke RE. Theory and applications of the Delphi technique: A bibliography (1975-1994). Technol Forecasting Soc Change. 1996;53(2):185-211.

24. Burt C. Proceso de Evaluación Rápido (RAP) y Comparación con el Patrón de Referencia (benchmarking). ITRC Report No. R 01-009. San Luis Obispo, CA: California Polytechnic State University; 2001. Disponible: http://www.itrc.org/reports/pdf/ rapespanol.pdf Acceso el 14 de abril de 2018.

25. Karolinski A, Mercer R, Salgado P, Ocampo C, Bolzán A, Nieto R, et al. Primer informe nacional de relevamiento epidemiológico SIP-Gestión. Desarrollo e implementación a escala nacional de un sistema de información en salud de la mujer y perinatal en Argentina. Buenos Aires: Ministerio de Salud; 2013. Disponible en: http:// publicaciones.ops.org.ar/publicaciones/ publicaciones $\% 20$ virtuales /SIP-G_ Anexos/pdfPublicacion/SIP-G.pdf Acceso el 14 de abril de 2018.

26. Ministerio de Salud. Programa Sumar. Buenos Aires: Ministerio de Salud; 2018. Disponible en: http:/ /www.msal.gob.ar/ sumar/index.php/institucional/programa-sumar-mas-salud-publica Acceso el 14 de abril de 2018.

27. Mainero L, Martínez G, Rubino M, De Mucio B, Díaz Rosello JL, Durán P, et al. Sistema informático perinatal (SIP): manual de uso del programa para el análisis y aprovechamiento de la información. 2a. ed. Montevideo: CLAP/SMR; 2011. (Publicación científica 1587.)

28. Dirección Nacional de Maternidad e Infancia. SIP-GESTIÓN: Manual operativo para su implementación en 100
Maternidades Públicas y Sistema de Obras Sociales Provinciales de la Argentina. Buenos Aires: Dirección Nacional de Maternidad e Infancia; 2012. Disponible en: http: / / publicaciones.ops.org.ar / publicaciones / publicaciones \%20virtuales/SIP-G_Anexos/otrasPub / 01.\%20 Publicaciones \%20del\%20Ministerio\%20 $\mathrm{d}$ e $\% 20 \mathrm{~S}$ a 1 u d $\% 20 \mathrm{de} \% 201$ a $\% 20$ Naci\%C3\%B3n/07.\%20Manual\%20SIP-G. pdf Acceso el 14 de abril de 2018.

29. World Health Organization. Coverage and maternal care. A listing of available information. Fourth ed. Geneva: WHO; 1997.

30. Payne P. Appropriate indicators for Project design and evaluation. UNICEF/WFP Workshop on food aid and well-being of children in the developing World. New York: UNICEF; 1985.

31. Donabedian A. Explorations in quality assessment and monitoring. Vol 1. The definition on quality and approaches to it assessment. Ann Arbor, MI: Health Administration Press; 1980.

32. Zeitlin J, Wildman K. PERISTAT. Indicators for monitoring and evaluating perinatal health in Europe. Scientific final report. Health Monitoring Programme. Brussels: European Union; 2000. Disponible en: https://ec.europa.eu/health/ph_projects/2000/monitoring/fp_monitoring_2000_frep_07_en.pdf Acceso el 10 de agosto de 2018.

33. Perinatal Partnership Program of Eastern and Southeastern Ontario, Canada. Annual Perinatal Statistical Report 2003. Ottawa: Health Canada; 2003.

34. Ramos S, Romero M, Karolinski A, Mercer R, Insúa I, del Río Fortuna C. Mortalidad Materna en Argentina: Diagnóstico para la reorientación de políticas y programas de salud. Buenos Aires: CEDES; 2004. Disponible en: http:/ / www.cedes.org.ar/ Publicaciones /SSPP/2004/SSPP200402. pdf Acceso el 14 de abril de 2018.

35. Ministerio de Salud. Dirección de Estadísticas e Información de la Salud. Anuario 2004. Buenos Aires: Ministerio de Salud; 2004.

36. National Perinatal Epidemiology and Statistics Unit. Report on the evaluation of the Perinatal National Minimum Data Set. Sydney: NPESU; 2004.

37. Centro Latinoamericano de Perinatología y Desarrollo Humano. Sistema Informático Perinatal Indicadores Básicos Perinatales 2005. Montevideo: CLAP; 2005.

38. Centro Latinoamericano de Perinatología y Desarrollo Humano. Indicadores de la Federación Internacional de Ginecología y Obstetricia. Sistema Informático Perinatal (SIP/CLAP/PAHO/OMS). Montenivideo: CLAP; 2005.
39. Devane D1, Begley CM, Clarke M, Horey D, OBoyle C. Evaluating maternity care: a core set of outcome measures. Birth. 2007;34(2):164-72. Disponible en: https:// www.ncbi.nlm.nih.gov/pubmed/17542821 Acceso el 14 de abril de 2018.

40. Dawn P, Guyer B, Allston A. Integrated Perinatal Framework. A multiple determinants model with a life span approach. Am J Pev Med. 2003;25(1):67-75.

41. The Futures Group International. Networking for policy change: An advocacy training manual by POLICY. Washington, DC: The Futures Group International; 2002.

42. Center for Human Services. Client communication behavior with healthcare providers in Indonesia. Bethesda, MD: Center for human services; 2002. Disponible en: https: / / www.usaidassit.org/sites/assist/files/indonesia_client_communication_behaviors_pdf.pdf Acceso el 19 de septiembre de 2018.

43. Karolinski A, Mercer R, Micone P, Ocampo C, Salgado P, Corte V, et al. Bases para establecer un sistema de vigilancia activa y respuesta rápida para el manejo de la morbilidad materna severa. Rev Argent Salud Publica. 2015;6(23):7-14.

44. Karolinski A, Mazzoni A, Belizán JM, Althabe F, Bergel E, Buekens P. Lost opportunities for effective management of obstetric conditions to reduce maternal mortality and severe maternal morbidity in Argentina and Uruguay. Int J Gynaecol Obstet. 2010;110(2):175-80.

45. Karolinski A, Mercer R, Micone $\mathrm{P}$, Ocampo C, Mazzoni A, Fontana O, et al. The epidemiology of life-threatening complications associated with reproductive process in public hospitals in Argentina. Br J Obstet Gynecol. 2013;120(13):1685-95.

46. Ministerio deSalud, Gobierno de la Ciudad Autónoma de Buenos Aires. Resolución 1969 publicada en BO del 30122013. Uso obligatorio del Sistema Informático Perinatal. Buenos Aires: Gobierno de la Ciudad Autónoma de Buenos Aires; 2013. Disponible en Internet:http:/ / test.e-legisar.msal.gov.ar/leisref/public/showAct. php?id=23094\&word=sip Acceso el 14 de abril de 2018.

Manuscrito recibido el 15 de mayo de 2018. Aceptado para publicación, tras revisión, el 18 de julio de 2018. 
ABSTRACT

Foundations for the development and implementation of a women's and perinatal health information model for management in Latin America

Keywords
Objective. The Perinatal Information System (SIP) represents a milestone in the use of systematized information in the Region of the Americas. What has been learned from the system has contributed to the development of a model based on a set of core indicators (SCI). The objective of the study was to describe the historical and methodological process involved in the development, implementation, and territorial scaling-up of an SCI to monitor and evaluate women's and perinatal health policies, programs, and services geared to management (SIP-GESTIÓN).

Methods. The study was conducted in two stages: 1) a four-phase validation of an SCI in a hospital network: a) construction of the theoretical indicator model, b) operations research, c) final selection of the indicators, and d) the definition of reference standards; and 2) territorial scaling-up.

Results. A total of 17 indicator models were identified. The initial model included 177 indicators divided into seven dimensions (context, habits, accessibility, use of services, quality of care, maternal and fetal impact, and maternal and neonatal impact), with 21 indicators remaining after three Delphi rounds. The final model (SIPGESTIÓN), which included 40 indicators, was then used to study $240,021(79.1 \%)$ of the 303,559 deliveries attended in 122 selected maternity facilities in 24 jurisdictions $(100 \%)$ in Argentina. The information is presented in national terms and by health region, province, and hospital.

Conclusions. This model has made it possible to achieve high levels of information coverage and quality and territorial scaling-up and is useful for management, research, and the reorientation of programs and policies.

Health information management; scientific information; health decision making; information systems; women's health; perinatology; Latin American Center for Perinatology; women and reproductive health; Argentina.
RESUMO

Fundamentos do desenvolvimento e implementação de um modelo de informação em saúde da materna e perinatal orientado à gestão na América Latina

Palavras-chave
Objetivo. O Sistema de Informação Perinatal (SIP) é um marco no uso de informação sistematizada na Região das Américas. A experiência obtida contribuiu para o desenvolvimento de um modelo baseado em um conjunto mínimo de indicadores (CMI). $\mathrm{O}$ objetivo do estudo foi descrever o processo histórico e metodológico do desenvolvimento, implementação e dimensionamento territorial do CMI para monitorar e avaliar políticas, programas e serviços de saúde materna e perinatal orientado à gestão (SIP-gestão).

Métodos. O estudo foi realizado em duas etapas. A primeira etapa consistiu da validação em quatro fases de um CMI em uma rede de hospitais: a) construção do modelo teórico de indicadores, b) implementação da pesquisa operacional, c) seleção final dos indicadores e d) definição dos padrões de referência. A segunda etapa consistiu da determinação da escala territorial.

Resultados. Foram identificados 17 modelos de indicadores. O modelo inicial incluiu 177 indicadores agrupados em sete dimensões (contexto, hábitos, acessibilidade, utilização de serviços, qualidade do atendimento, impacto materno-fetal e impacto materno-neonatal) que foram reduzidas a 21 após três rodadas de aplicação da técnica Delphi. O modelo final (SIP-gestão) inclui 40 indicadores. Foram analisados 240.021 partos $(79,1 \%)$ de um número total de 303.559 casos atendidos nas 122 maternidades selecionadas em 24 jurisdições (100\%) da Argentina. Os dados são apresentados ao nível nacional e desagregados por região de saúde, província e hospital. Conclusões. O modelo desenvolvido atingiu altos níveis de cobertura e qualidade da informação e determinação da escala territorial, e pode ser usado na gestão, pesquisa e reorientação de programas e políticas.

Gestaô da informaçaô em saúde; informaçaô científica; tomada de decisôes; sistemas de informaçâo; saúde da mulher; perinatologia; Centro Latinoamericano de Perinatologia; saúde da mulher e reprodutiva; Argentina. 